\title{
FoxP3-Positive T-Regulatory Cells in Lymph Nodes with Mycosis Fungoides and Sézary Syndrome
}

\author{
Rekha Bhat, ${ }^{1}$ Bhavna Khandpur, ${ }^{1}$ Eric C. Vonderheid, ${ }^{2}$ and J. Steve Hou ${ }^{1}$ \\ ${ }^{1}$ Department of Pathology, Drexel University College of Medicine, 245 N. 15th Street, Mail Stop 435, Philadelphia, PA 19102, USA \\ ${ }^{2}$ Johns Hopkins Medical Institutes, Baltimore, MD 21287, USA \\ Correspondence should be addressed to Rekha Bhat; r.balkur@gmail.com
}

Received 27 July 2014; Revised 19 October 2014; Accepted 21 October 2014; Published 11 November 2014

Academic Editor: Jan Delabie

Copyright (c) 2014 Rekha Bhat et al. This is an open access article distributed under the Creative Commons Attribution License, which permits unrestricted use, distribution, and reproduction in any medium, provided the original work is properly cited.

\begin{abstract}
Mycosis fungoides and Sézary syndrome are indolent cutaneous T-cell lymphomas, with skin-associated peripheral lymph nodes being the most frequent extracutaneous site of involvement. Acquisition of functional properties of regulatory T-cells by malignant T-cells in advanced disease may contribute to immunosuppression. Whereas previous studies examining FoxP3 protein expression in mycosis fungoides and Sézary syndrome have focused on skin specimens, little data are available on lymph nodes from patients with these conditions. In this study we examined FoxP3+ regulatory T-cells in lymph nodes from 26 patients with mycosis fungoides and Sézary syndrome and correlated the findings with clinical data, molecular assays for T-cell clonality, and flow cytometry. Except for one case of Sézary syndrome in which malignant T-cells expressed FoxP3 protein, a significantly lower number of FoxP3-expressing cells occurred in lymph nodes that were clearly involved with lymphoma as compared to uninvolved nodes. Cox proportional hazards model showed that lymph node rating and histological evidence of transformation, but not number of FoxP3+ cells, were factors significantly associated with adverse prognosis. We speculate that modulation of FoxP3+ cells in lymph nodes involved with lymphoma might play a role in disease progression. Attainment of a regulatory T-cell phenotype by a subset of lymphoma cells might signal a poor prognosis.
\end{abstract}

\section{Introduction}

Mycosis fungoides (MF) and Sézary syndrome (SS) are indolent cutaneous T-cell lymphomas (CTCL) with an approximately $90 \% 5$-year survival rate for MF and $20 \%$ to $40 \%$ survival rate for SS, depending on the criteria used to define SS $[1,2]$. Skin-associated peripheral lymph nodes (LNs) are the most frequent extracutaneous site of involvement in MF/SS, and the degree of histological evidence of involvement defines the $\mathrm{N}$ rating in the current clinical staging classification for $\mathrm{MF} / \mathrm{SS}$ based on tumor-node-metastasis-blood criteria [3]. In addition to $\mathrm{N}$ rating and clinical stage, prognosis is impacted in MF/SS by histological transformation to aggressive largecell lymphoma [4-6].

The malignant T-cells found in most cases of MF/SS have a CD4+, CD45RO+ phenotype with aberrant expression of Tcell-associated antigens, including loss of CD7 and/or CD26 [1, 7-11]. Because malignant T-cells are capable of producing $\mathrm{T}_{\mathrm{H}} 2$ cytokines (IL-4, IL-5, and IL-10), it has been suggested that, with advancing disease, these cytokines modulate the host antitumor immune response toward tolerance $[12,13]$. However, recent evidence suggests that malignant T-lymphocytes in advanced disease may also acquire functional properties of regulatory $\mathrm{T}$ (Treg) cells that can also contribute towards immunosuppression [14-17].

Normal Treg cells, identified in tissue sections by the transcription factor FoxP3, have been invested with a multitude of roles in immune regulation. An increased number of Treg cells in tumor infiltrates, particularly solid tumors, are often associated with poor prognosis [18, 19]. Conversely, an increase in normal FoxP3+ Treg cells in dermal infiltrates of MF has been correlated with a favorable prognosis, similar to that of other indolent lymphomas [20]. However, if neoplastic T-cells of SS express FoxP3 mRNA, the prognosis may be relatively unfavorable [21].

Previous studies examining FoxP3 protein expression in MF/SS have focused mostly on skin biopsy specimens. A single study examined FoxP3+ cells in 9 LNs [22]. We 
TABLE 1: Histological classification of lymph nodes.

\begin{tabular}{lccccc}
\hline Diagnostic group & LN0-1 & LN2 & LN4 & DL & CTCL \\
\hline MF & 8 & 2 & 3 & $\mathbf{1 0}$ & $\mathbf{3}$ \\
Patch & 2 & 1 & 1 & $\mathbf{3}$ & $\mathbf{1}$ \\
Plaque & 3 & 1 & 0 & $\mathbf{4}$ & $\mathbf{0}$ \\
Tumor & 3 & 0 & 2 & $\mathbf{3}$ & $\mathbf{2}$ \\
E-CTCL & 3 & 2 & 8 & $\mathbf{5}$ & $\mathbf{8}$ \\
E-MF & 0 & 1 & 0 & $\mathbf{1}$ & $\mathbf{0}$ \\
SS & 0 & 0 & 7 & $\mathbf{0}$ & $\mathbf{7}$ \\
NOS & 3 & 1 & 1 & $\mathbf{4}$ & $\mathbf{1}$ \\
\hline All cases & 11 & 4 & 11 & $\mathbf{1 5}$ & $\mathbf{1 1}$ \\
\hline
\end{tabular}

DL: dermatopathic lymphadenopathy; CTCL: cutaneous T-cell lymphoma; E: erythrodermic; MF: mycosis fungoides; SS: Sézary syndrome; NOS: not otherwise specified.

examined FoxP3+ Tregs in LN specimens from patients with $\mathrm{MF} / \mathrm{SS}$ (including both involved and uninvolved LNs) and correlated the findings with clinical data, molecular assays for T-cell clonality, and flow cytometry.

\section{Materials and Methods}

Tissue blocks from LN specimens obtained from 26 patients with confirmed MF or erythrodermic CTCL including SS, between January 1991 and August 2001, were used to construct a tissue microarray. Histological classification of excised LN specimens was based on NCI-MFCG criteria [23] with LN grade 4 (LN4), defined as partial or complete effacement of nodal architecture by atypical cells, signifying prognostically significant nodal involvement based on prior experience [24] and recent recommendations of the International Society of Cutaneous Lymphomas/European Organization of Research and Treatment of Cancer [3]. Histological grades LN0-LN2 were considered to represent dermatopathic lymphadenopathy (DL) with isolated or small clusters of atypical cells (one case had no atypical cells/LN0). None of the cases showed an LN3 pattern. Accordingly, the cohort was divided into two groups for analysis: 15 cases with DL (LN0-2) and 11 cases with CTCL (LN4) in LN specimens (Table 1).

Immunohistochemical staining using antibodies to FoxP3, CD3, CD4, and CD30 (see Table 2 for antibody sourcing and dilutions) was performed according to established methods. FoxP3+ CD4+ cells in the interfollicular T-zone of the LNs were counted within a $1-\mathrm{mm}^{2}$ grid under magnification $\times 400$. Positive staining with FoxP3 was observed to be nuclear in all cases. This pattern of staining has been reported in activated T-cells but not in resting T-cells [25].

Flow cytometry using commercially available monoclonal antibodies that reacted against pan-T-cell-associated markers CD3, CD4, and CD8 was performed on 24 specimens, against CD7 on 23 specimens, and against CD25 on 21 specimens. The analysis involved individual antibodies before July 1997 and dual antibodies (e.g., CD4+CD7- subset) thereafter.
TABLE 2: Antibodies and dilutions used in the study.

\begin{tabular}{lcc}
\hline Antibody & Manufacturer & Dilution \\
\hline CD3 & Dako, Carpinteria, CA & $1: 100$ \\
CD4 & Dako, Carpinteria, CA & $1: 10$ \\
CD30 & Dako, Carpinteria, CA & $1: 50$ \\
Foxp3 (236A/E7) & Abcam, Cambridge, MA & $1: 25$ \\
\hline
\end{tabular}

Molecular genetic analysis for rearrangement of the T-cell receptor gamma gene was performed in reference laboratories on original material using the polymerase chain reaction(PCR-) denaturing gradient gel electrophoresis (20 patients) and the PCR-single-strand conformation polymorphism techniques (patients 2 and 19). Patient 22 had a clone demonstrated by Southern blot analysis of the T-cell receptor beta gene. PCR with fluorophore-labeled primers and capillary gel electrophoresis was used to study an additional 3 cases (patients 9, 10, and 21) not initially studied as well as to confirm results on 7 specimens studied by other PCR methods.

Results of laboratory studies were given as median value with range. The Mann-Whitney test was used to determine differences in laboratory test values for two groups classified according to LN involvement. Fisher's and Pearson's chi square exact tests were used to test categorical data in 2 by 2 and $\mathrm{R}$ by $\mathrm{C}$ tables, respectively. The Cox proportional hazards model was used to examine the relationship between prognostic factors and survival. Survival was determined from the time of the LN biopsy to the date of death (uncensored observation) or contact as of October 2014 (censored observation). Death from any cause used to define overall survival and death caused by CTCL or its treatment including sepsis was used to define CTCL-related survival. The statistical packages used for data analysis and graphs were SYSTAT 10 and SPSS 13.0 for Windows (IBM SPSS, Chicago, IL); StatXact 3 (Cytel, Cambridge, MA); and SigmaPlot 9.0, (Systat Software, Point Richman, CA).The study was carried out after obtaining institutional ethics committee approval (exempt).

\section{Results}

The clinical diagnosis at the time of biopsy was patch phase MF ( 4 cases), plaque phase MF (4 cases), tumor phase MF (5 cases), erythrodermic MF (1 case), SS (7 cases), and erythrodermic CTCL, not otherwise specified (5 cases). The clinical features are shown in Table 3.

3.1. Flow Cytometry. Although flow cytometry showed a higher percentage of CD4+ cells and lower percentage of $\mathrm{CD} 8+$ that resulted in a higher $\mathrm{CD} 4 / \mathrm{CD} 8$ ratio in CTCL involved LNs compared to DL, the differences were not statistically significant (Table 4). In addition, no difference was apparent for the percentage of CD7+ cells, a T-cell marker often decreased or lost by neoplastic cells of CTCL $[26,27]$ and CD25+ cells, which represent both activated T- and Treg cells. However, the calculated CD4/CD7 ratio was significantly higher in involved LNs than in DL $(P=0.035)$. 
TABLE 3: Clinical features of cases included in the study.

\begin{tabular}{|c|c|c|c|c|c|}
\hline Diagnostic group & Number of cases & $\mathrm{M} / \mathrm{F}$ & Median age, years (range) & Follow-up (months) & Number of deaths $(\%)^{\mathrm{a}}$ \\
\hline $\mathrm{MF}$ & 13 & $6 / 7$ & $68(35-86)$ & $87.3(0.7-270.3)$ & $7(54)$ \\
\hline Patch & 4 & $3 / 1$ & $69(64-86)$ & $119.9(12.3-157.9)$ & $1(25)$ \\
\hline Plaque & 4 & $1 / 3$ & $69(35-74)$ & $116.4(27.5-155.1)$ & $3(75)$ \\
\hline Tumor & 5 & $2 / 3$ & $63(35-74)$ & $18.4(0.7-270.3)$ & $3(60)$ \\
\hline E-CTCL & 13 & $7 / 6$ & $69(30-84)$ & $62.1(0.8-244.8)$ & $8(62)$ \\
\hline E-MF & 1 & $1 / 0$ & 67 & 185.3 & $0(0)$ \\
\hline SS & 7 & $4 / 3$ & $71(30-84)$ & $19.8(0.8-62.1)$ & $7(100)$ \\
\hline NOS & 5 & $2 / 3$ & $61(45-83)$ & $127.6(80.4-244.8)$ & $1(20)$ \\
\hline All cases & 26 & $13 / 13$ & $68(30-86)$ & $71.3(0.7-270.3)$ & $15(58)$ \\
\hline
\end{tabular}

${ }^{a}$ Death from disease-related cause.

M: male; F: female; E: erythrodermic; CTCL: cutaneous T-cell lymphoma; MF: mycosis fungoides; SS: Sézary syndrome; NOS: not otherwise specified.

TABLE 4: Flow cytometry, immunohistochemistry, and T-cell clonality results from lymph nodes.

\begin{tabular}{|c|c|c|c|}
\hline $\begin{array}{l}\text { Clinical } \\
\text { diagnosis }\end{array}$ & $\mathrm{DL}(n=15)$ & CTCL $(n=11)$ & $P$ value \\
\hline $\mathrm{MF}(\mathrm{PT} / \mathrm{PQ} / \mathrm{T})$ & $3 / 4 / 3$ & $1 / 0 / 2$ & 0.720 \\
\hline $\begin{array}{l}\text { E-CTCL } \\
(\text { EMF/NOS/SS) }\end{array}$ & $1 / 4 / 0$ & $0 / 1 / 7$ & 0.005 \\
\hline \multirow[t]{2}{*}{ CD3 } & $(N=13)$ & $(N=11)$ & \multirow{2}{*}{0.772} \\
\hline & $70(46-94)$ & 67 (24-96) & \\
\hline \multirow[t]{2}{*}{ CD4 } & $(N=13)$ & $(N=11)$ & \multirow{2}{*}{0.098} \\
\hline & $55(21-81)$ & 66 (15-98) & \\
\hline \multirow[t]{2}{*}{$\mathrm{CD} 8$} & $(N=13)$ & $(N=11)$ & \multirow{2}{*}{0.210} \\
\hline & $8(2-16)$ & $5(2-23)$ & \\
\hline \multirow[t]{2}{*}{$\mathrm{CD} 4 / 8$} & $(N=13)$ & $(N=11)$ & \multirow{2}{*}{0.173} \\
\hline & $6.8(2.4-34.5)$ & $8.8(2.7-49.0)$ & \\
\hline \multirow[t]{2}{*}{$\mathrm{CD} 7$} & $(N=13)$ & $(N=10)$ & \multirow{2}{*}{0.154} \\
\hline & $50(28-82)$ & $34(10-93)$ & \\
\hline \multirow[t]{2}{*}{$\mathrm{CD} 4 / 7$} & $(N=13)$ & $(N=10)$ & \multirow{2}{*}{0.035} \\
\hline & $1.0(0.4-2.5)$ & $1.7(0.9-8.8)$ & \\
\hline \multirow[t]{2}{*}{$\mathrm{CD} 3 / 7$} & $(N=13)$ & $(N=10)$ & \multirow{2}{*}{0.172} \\
\hline & $1.2(0.9-2.6)$ & $2.1(1.0-9.0)$ & \\
\hline \multirow[t]{2}{*}{ CD25 } & $(N=12)$ & $(N=9)$ & \multirow{2}{*}{0.593} \\
\hline & $9(2-34)$ & $7(0.1-34)$ & \\
\hline \multirow{2}{*}{$\begin{array}{l}\text { FoxP3 + } \\
\left(\text { cells } / \mathrm{mm}^{3}\right)\end{array}$} & $(\mathrm{N}=15)$ & $(\mathrm{N}=11)$ & \multirow{2}{*}{$<0.001$} \\
\hline & 99 (64-257) & $32(0-189)$ & \\
\hline T-cell clonality & $5(33)$ & $8(73)$ & 0.111 \\
\hline
\end{tabular}

E: erythrodermic; CTCL: cutaneous T-cell lymphoma; MF: mycosis fungoides; SS: Sézary syndrome; NOS: not otherwise specified; patch (PT), plaque (PQ), and tumor $(\mathrm{T})$ phases.

It is worth noting that a strong positive correlation (rho $=0.905, P<0.001)$ was present between the calculated CD4/CD7 ratio and the percentage of CD4+CD7- cells for 8 LNs studied simultaneously with two antibodies.

3.2. Immunohistochemical Staining for FoxP3. A significantly lower number of FoxP3-expressing cells occurred in LNs that were clearly affected by CTCL (median, 32 cells $/ \mathrm{mm}$, range, 0-189 cells $/ \mathrm{mm}^{3}$ ) compared to DL (median, 99 cells $/ \mathrm{mm}^{3}$, range, $64-257$ cells $/ \mathrm{mm}^{3}$; Tables 4 and 5, Figures $1(\mathrm{a})-1(\mathrm{~d})$ ). However, when FoxP3 numbers were plotted according to histological diagnosis (Figure 2), one specimen, obtained from a Sézary patient (case 24), had an unusually high number of FoxP3+ cells. This patient clinically had a sudden and rapid increase in cervical and other LNs after a 4-year interval of stable untreated disease. Large cell transformation was suspected, but the specimen showed a diffuse mixed cell pattern. DNA cell cycle analysis showed a diploid DNA index with an S-phase of 8.3\%. immunohistochemistry and flow cytometry confirmed that the neoplastic cells were $\mathrm{CD} 2+3+4+5+7+8-\mathrm{HLADR}+(\mathrm{CD} 4 / \mathrm{CD} 8$ ratio $92 \% / 2 \%)$ with most cells also expressing other activation markers, that is, CD71+, CD38, and CD25. The histoimmunopathological findings did not fulfill the published criteria for transformed MF [4, 28, 29]. FoxP3 was expressed by both morphologically normal and neoplastic cells (Figures 1(e) and 1(f)). In addition, CD30 staining revealed only scattered large CD30+ cells $(14 \%-16 \%$ of cells, Figure $1(\mathrm{~g}))$. This finding supports the prevailing concept that FoxP3 is typically not expressed by neoplastic T-cells in most cases of CTCL [30].

3.3. Cox Proportional Hazards Analysis. When analyzed in the Cox model for prognostic implications, a number of variables correlated significantly to prognosis (disease-related deaths). These included categorization into DL versus CTCL groups $(P=0.001)$, LN rating $(P=0.003)$, histologic evidence of large-cell transformation $(P=0.003)$, molecular evidence of T-cell clonality $(P=0.002)$, and the CD4/CD8 ratio $(P=0.035)$. Variables that were not significantly correlated with prognosis were patients' age $(P=0.219)$, skin T rating $(P=0.69)$, maximum LN size $(P=0.686)$, and calculated CD4/CD7 ratio $(P=0.072)$. Notably, the number of FoxP3+ cells $/ \mathrm{mm}^{3}$ also did not have prognostic importance $(P=$ 0.109 ) when considered for the entire dataset. However, if the outlier Sézary patient with aberrant expression of FoxP3 and aggressive disease (case 24) was excluded from the analysis, the density of FoxP3+ cells became significant $(P=0.04)$ for the remaining cases. This finding is expected because of the strong inverse correlation between histopathologic 


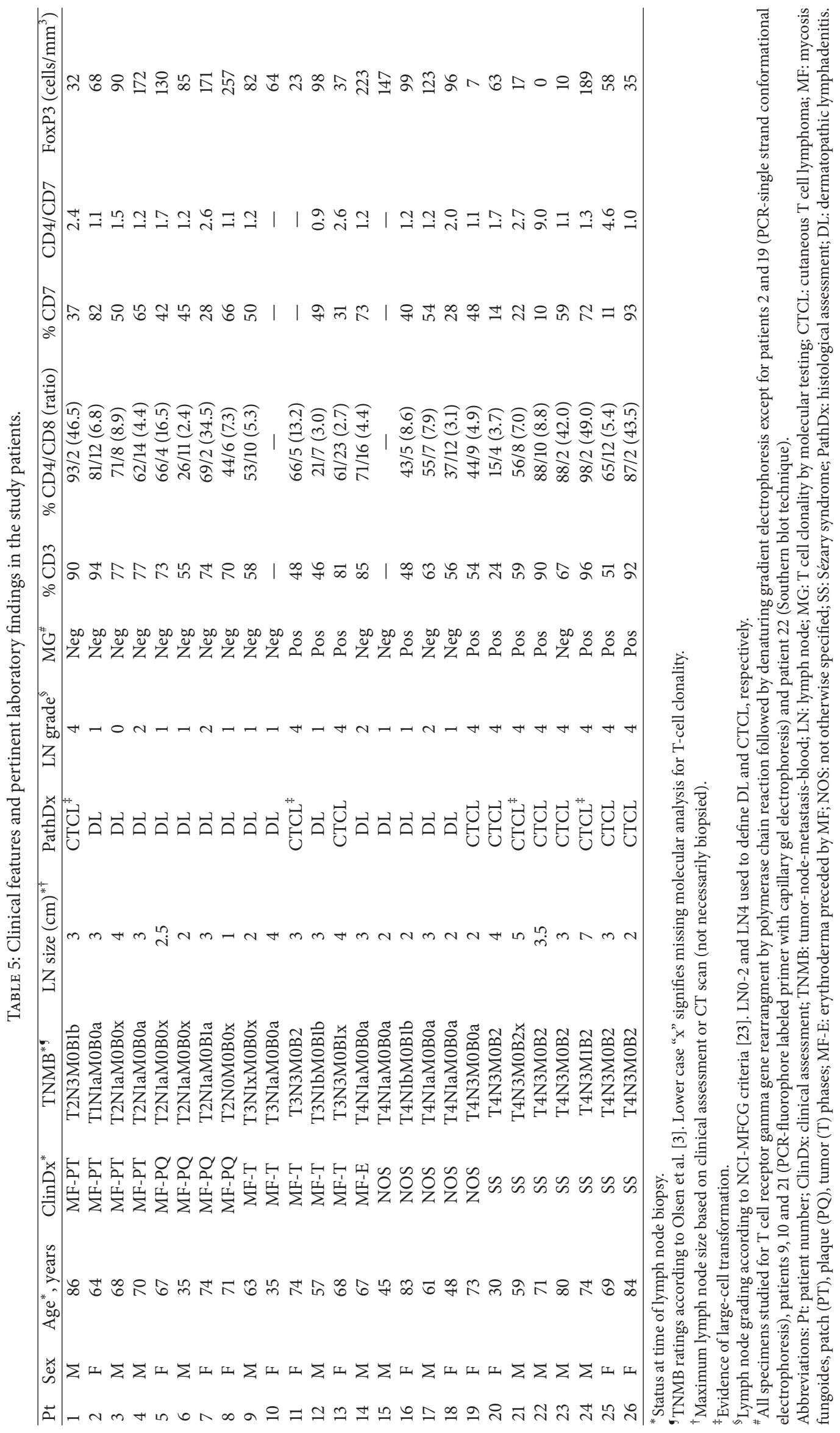




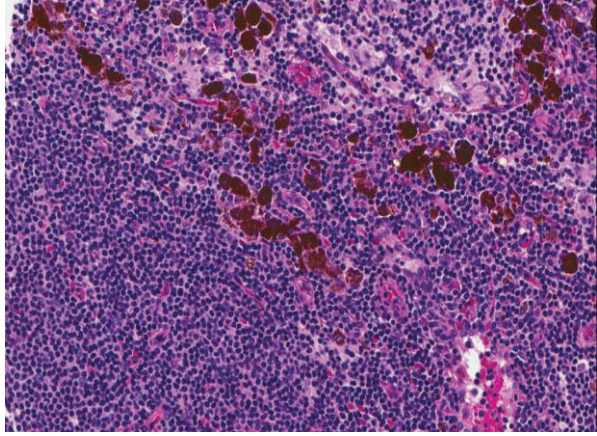

(a)

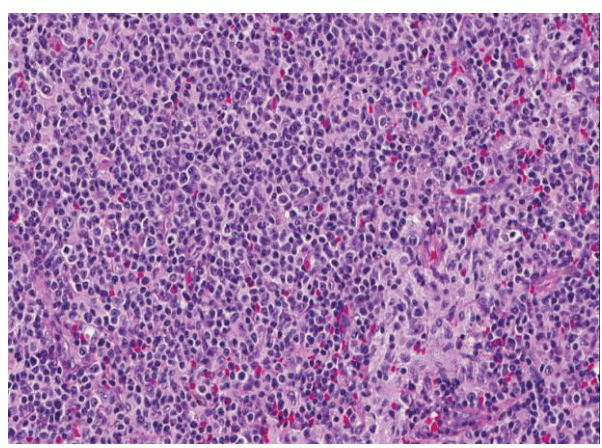

(c)

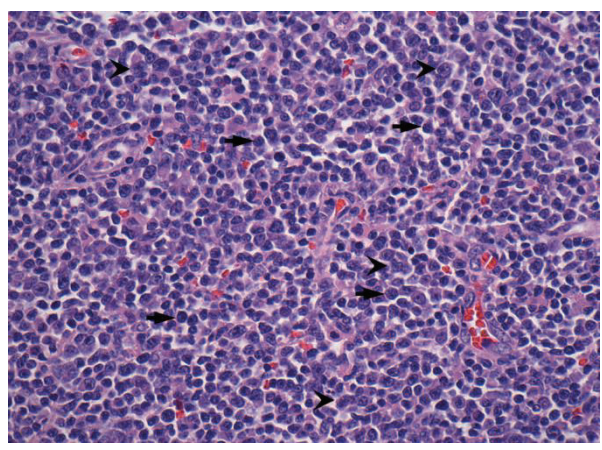

(e)

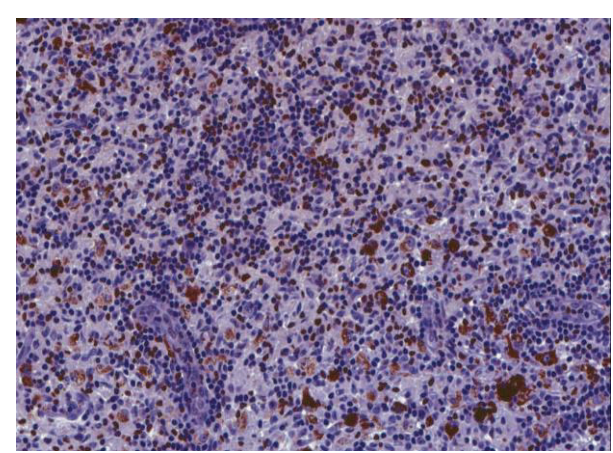

(b)

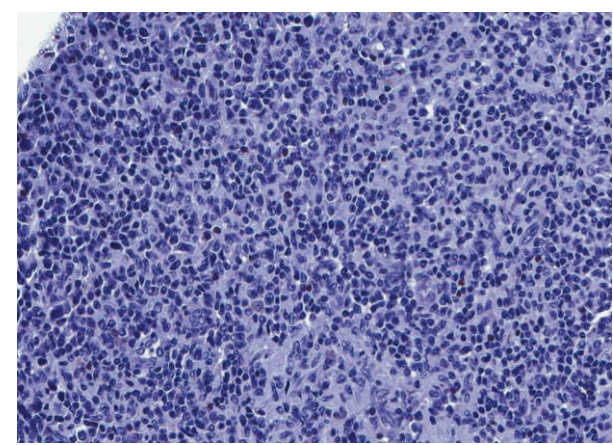

(d)

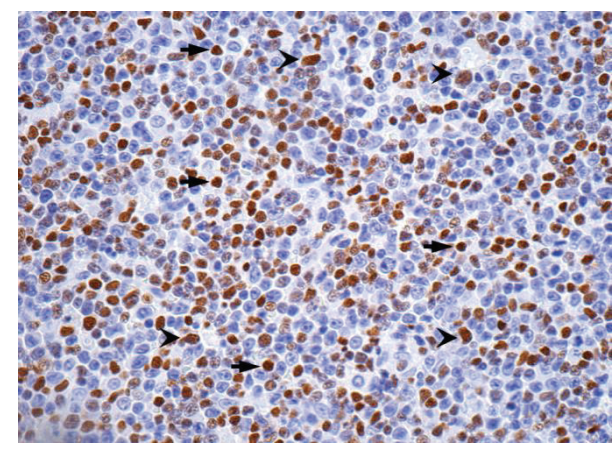

(f)

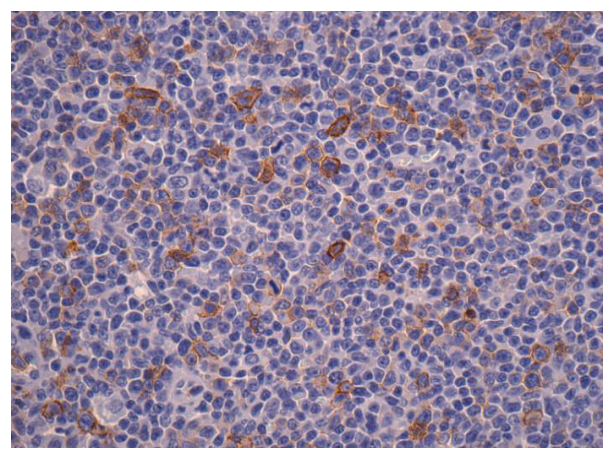

(g)

Figure 1: (a) Hematoxylin \& eosin- and (b) anti-FoxP3-stained sections of lymph node with dermatopathic lymphadenopathy. (c) Hematoxylin \& eosin- and (d) anti-FoxP3-stained sections of lymph node with diffuse CTCL. (e-g) Photomicrographs of sections from case 24 ( $\times 400)$. (e) Hematoxylin \& eosin-stained section demonstrating large cerebriform (arrowheads) and small noncerebriform (arrows) lymphocytes; (f) anti-FoxP3-stained section demonstrating positive cells with large cerebriform (arrowheads) and small noncerebriform (arrows) lymphocytes; (g) CD30-stained section demonstrating scattered large cells. 


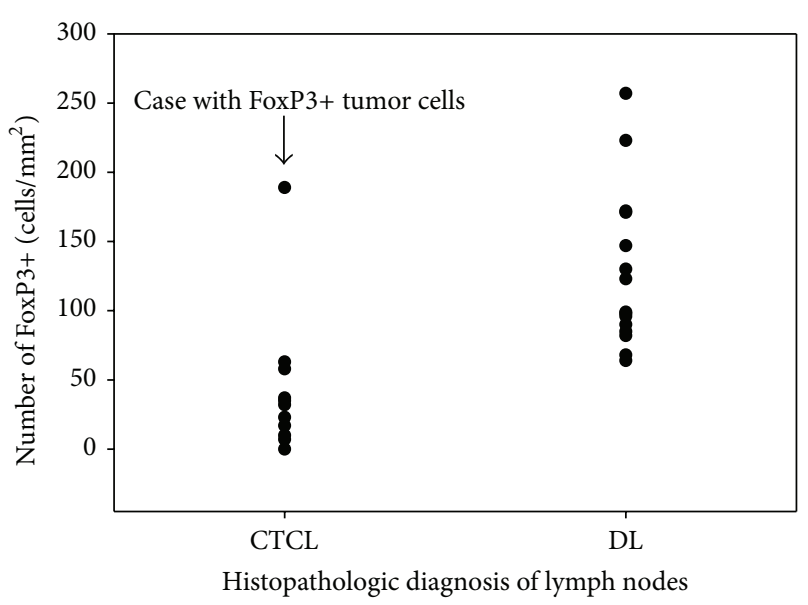

FIgURE 2: Scatter plot of FoxP3-positive cell counts $/ \mathrm{mm}^{2}$ against histopathologic diagnosis.

involvement by CTCL and low FoxP3+ cell numbers in LNs (Figure 2). Indeed, when both histopathologic diagnosis (CTCL versus DL) and FoxP3 percentages are analyzed in the Cox model, only histopathologic diagnosis retains significance.

Considering that the neoplastic T-cells of MF/SS typically do not express FoxP3 and FoxP3+ Tregs infiltrating skin lesions of MF appear to have a beneficial influence on prognosis, we wondered if a low percentage of FoxP3 + cells might signify a worse prognosis for patients without histopathologic involvement of LNs. However, in our 16 cases of DL, the number of FoxP3+ cells did not correlate with disease-related death $(P=0.553)$. Of the other variables, only molecular evidence of T-cell clone correlated with prognosis in this subset $(P=0.021)$.

Likewise with the one exception of the patient with aggressive Sézary syndrome (case 24), the percentage of FoxP3+ cells in LNs involved by CTCL did not appear to correlate with adverse prognosis. In addition, of 11 cases with effaced LN architecture (LN4), the percentage of FoxP3+ cells was not significantly different for the 3 cases with evidence of large cell transformation (median, 23\%, range, 17 to $32 \%$ ) compared to 8 cases without transformation (median, $36 \%$, range, 0 to $189 \% ; P=0.54$ ). The same conclusion was also reached if case 24 was excluded (median, 35\%, range, 0 to $63 \% ; P=0.73$ ). None of the other variables provided prognostic information for this subset of cases.

\section{Discussion}

Krejsgaard et al. recently reviewed the complex relationship between FoxP3-expressing tumor-infiltrating Treg cells and neoplastic T-cells in MF and SS [31]. In summary, normal FoxP3+ Tregs are readily detectable in the dermal infiltrates of clinically early patch or plaque lesions of MF at numbers the same as or slightly less than common inflammatory skin diseases [20, 22, 30, 32-34]. Except for one study that compared FoxP3 expression in patch/plaque lesions versus tumors in the same patients [35], most studies found that the number of Tregs is lower in MF tumors compared to patch/plaques including tumors with large cell transformation $[20,22,34]$. Gjerdrum also reported that number of Tregs had prognostic significance that was independent of the morphologic groupings in MF [20]. This finding supports the concept that Treg cells inhibit expansion of the neoplastic Tcell population.

These immunohistochemical studies also indicate that neoplastic T-cells of MF typically do not express FoxP3 [20, $25,30,34,36]$. However, in some cases, some neoplastic cells in the epidermis of patch/plaque MF lesions or the dermal infiltrate of tumors express FoxP3 [20, 22, 35, 37]. The level of FoxP3 expression is often weaker than Treg cells [20]. Some of the disparate results in these studies may be due to methodological differences or the difficulty in distinguishing benign from neoplastic T-cells in tissue sections [35].

As with advanced MF lesions, the number of normal Tregs in the cutaneous infiltrates of SS is low and FoxP3+ neoplastic T-cells are usually but not always absent [16, 17, 30, 38]. Flow cytometry also has shown variable expression levels of FoxP3 protein by circulating neoplastic T-cells [17, 38-40]. Recently, Heid et al. proposed that SS can be divided into two subsets based on low or high expression of FoxP3 and this is reflected in the dermal infiltrates of these patients [17]. The Sézary cases with relatively high FoxP3 expression levels by circulating neoplastic cells compared to controls had relatively high proportion of FoxP3+ cells in the skin. Although CD25, a marker of normal Treg cells, was usually absent on FoxP3+ neoplastic cells, these cells displayed a suppressive effect on cell proliferative in vitro and the FOXP3 gene locus was found to be highly demethylated similar to naturally occurring Treg cells. Thus, the possibility that some cases of SS are in fact malignancies of Treg cells as originally proposed by Berger et al. exists [14, 41].

The prognostic implications of FoxP3 expression in SS are uncertain due to the small number of cases studied. One might expect SS with Treg-like functional properties to have a worse prognosis compared to SS without Treg-like properties because of potentially greater immunosuppressive effects on natural immune defenses. However, Heid et al. observed no difference in clinical and laboratory findings between the low and high FoxP3-expressing cases and suggested a trend toward better prognosis for the high FoxP3 subset [17]. Conversely, Capriotti et al. observed that 6 of 25 Sézary cases with high expression of FoxP3 mRNA levels had a worse prognosis [21]. Considering that levels of FoxP3 mRNA were higher in Heid's high FoxP3-expressing cases than the low FoxP3-expressing case [17], it seems likely that the cases expressing relatively high FoxP3 mRNA in Capriotti's series correspond to the high FoxP3 subset. Further studies are required to determine the prognostic implications of FoxP3 mRNA and protein expression in patients with SS.

In this study, we examined the expression and prognostic significance of FoxP3 in LNs obtained from patients with MF/SS. Our findings concur with previous studies that show Tregs to be present in both reactive and involved LNs [22, 25]. Our results also show, with one notable exception, that FoxP3 expression is generally not expressed by neoplastic T-cells in LNs that are partially or completely effaced by CTCL 
including 3 cases with large cell transformation. In this regard, one previous study found that FoxP3 protein was expressed on less than $10 \%$ of atypical cells in LNs involved with CTCL and less than $5 \%$ of cases with large cell transformation [22].

Although an increased number of Tregs in skin lesions of MF have been reported to have a favorable prognosis that is independent of lesion type [20], we found no correlation with disease-related survival in dermatopathic LNs obtained from CTCL patients with relatively advanced skin involvement. However, we cannot exclude the possibility that Treg density might have prognostic value in LNs obtained from patients with clinically earlier MF or LNs classified as LN3. Moreover, because FoxP3 was not expressed by neoplastic T-cells for all but one patient with clear-cut LN involvement (architectural effacement present), the number of total FoxP3+ cells is less than in dermatopathic LNs from these patients. Thus a very low FoxP3 density might signify a worse prognosis if considered as the only variable. However, this is not the situation in clinical practice and unless most neoplastic cells express FoxP3 as illustrated by one of our cases, no prognostic information is apparent vis-à-vis standard morphologic assessment.

The exceptional case with FoxP3+ neoplastic cells in his LN was obtained from a 74-year-old patient with SS who was followed for 4 years without treatment until he suddenly developed sudden and rapid enlargement of LNs in his neck and all other nodal areas and splenomegaly. His clinical manifestations were unusual compared to other patients with SS we have evaluated in several respects: (1) the skin eruption consisted of patchy erythema that would wax and wane in intensity and at times even appear to spontaneously clear with minimal pruritus; (2) peripheral LNs were not palpable until the terminal phase of his disease; and (3) he developed widespread tinea corporis due to Trichophyton tonsurans as well as several types of epithelial skin cancers. Most of the transformed tumor cells in his LN expressed CD25 in addition to FoxP3, a circumstance that occurred in $20 \%$ of Heid's high FoxP3 Sézary subset [17]. It is tempting to speculate that our patient may have had FoxP3-expressing SS from the outset, and if so, the presumed immunosuppressive effects of the FoxP3+ neoplastic T-cells might have attenuated the skin erythema and promoted the widespread tinea corporis. This idea was first suggested by Shiue in their case of "invisible" SS [40].

\section{Conclusions}

Our study examined the presence of FoxP3+ cells in LNs from patients with MF/SS. We have demonstrated that a significantly lower number of FoxP3-expressing cells occurred in most LNs that were clearly involved with lymphoma as compared to dermatopathic nodes. Cox proportional hazards model showed that LN rating and histological evidence of transformation, but not number of FoxP3 + cells, were factors significantly associated with adverse prognosis. We speculate that modulation of FoxP3-positive cells in LNs involved with lymphoma might play a role in disease progression.
Attainment of a Treg phenotype by a subset of lymphoma cells might signal a worse prognosis that needs confirmation by prospective studies.

\section{Conflict of Interests}

The authors declare that there is no conflict of interests regarding the publication of this paper.

\section{Authors' Contribution}

Rekha Bhat constructed the tissue microarray block, performed the experiment, and wrote the paper. Rekha Bhat and Bhavna Khandpur analyzed the immunohistochemical stains. Eric C. Vonderheid and J. Steve Hou are coprincipal investigators of the study, recruited the patients, and coordinated the research.

\section{Acknowledgments}

This work was supported by the Department of Pathology, Drexel University College of Medicine (DUCOM). The authors thank the Cancer Center at Hahnemann University Hospital, Sharon Cavone (H. T., DUCOM), and Tammy Haines (Hahnemann University Hospital) for IHC technical support. These findings were presented in part in poster form at the annual meeting of the United States and Canadian Academy of Pathology, 2010, at Washington DC.

\section{References}

[1] R. Willemze, E. S. Jaffe, G. Burg et al., "WHO-EORTC classification for cutaneous lymphomas," Blood, vol. 105, no. 10, pp. 3768-3785, 2005.

[2] P. T. Bradford, S. S. Devesa, W. F. Anderson, and J. R. Toro, "Cutaneous lymphoma incidence patterns in the United States: a population-based study of 3884 cases," Blood, vol. 113, no. 21, pp. 5064-5073, 2009.

[3] E. Olsen, E. Vonderheid, N. Pimpinelli et al., "Revisions to the staging and classification of mycosis fungoides and Sezary syndrome: a proposal of the International Society for Cutaneous Lymphomas (ISCL) and the cutaneous lymphoma task force of the European Organization of Research and Treatment of Cancer (EORTC)," Blood, vol. 110, no. 6, pp. 1713-1722, 2007.

[4] B. Vergier, A. de Muret, M. Beylot-Barry et al., "Transformation of mycosis fungoides: clinicopathological and prognostic features of 45 cases," Blood, vol. 95, no. 7, pp. 2212-2218, 2000.

[5] E. Barberio, L. Thomas, F. Skowron, B. Balme, and S. Dalle, "Transformed mycosis fungoides: clinicopathological features and outcome," British Journal of Dermatology, vol. 157, no. 2, pp. 284-289, 2007.

[6] S. O. Arulogun, H. M. Prince, J. Ng et al., "Long-term outcomes of patients with advanced-stage cutaneous T-cell lymphoma and large cell transformation," Blood, vol. 112, no. 8, pp. 30823087,2008

[7] M. Kuchnio, E. A. Sausville, E. S. Jaffe et al., "Flow cytometric detection of neoplastic $\mathrm{T}$ cells in patients with mycosis fungoides based on levels of T-cell receptor expression," American Journal of Clinical Pathology, vol. 102, no. 6, pp. 856-860, 1994. 
[8] J. Edelman and H. J. Meyerson, "Diminished CD3 expression is useful for detecting and enumerating Sézary cells," American Journal of Clinical Pathology, vol. 114, no. 3, pp. 467-477, 2000.

[9] M. G. Bernengo, M. Novelli, P. Quaglino et al., "The relevance of the CD4+ CD26- subset in the identification of circulating Sezary cells," British Journal of Dermatology, vol. 144, no. 1, pp. 125-135, 2001.

[10] L. T. Washington, Y. O. Huh, L. C. Powers, M. Duvic, and D. Jones, "A stable aberrant immunophenotype characterizes nearly all cases of cutaneous T-cell lymphoma in blood and can be used to monitor response to therapy," BMC Clinical Pathology, vol. 2, article 5, 2002.

[11] W. G. Morice, J. A. Katzmann, M. R. Pittelkow, R. A. El-Azhary, L. E. Gibson, and C. A. Hanson, "A comparison of morphologic features, flow cytometry, TCR-V $\beta$ analysis, and TCR-PCR in qualitative and quantitative assessment of peripheral blood involvement by Sézary syndrome," The American Journal of Clinical Pathology, vol. 125, no. 3, pp. 364-374, 2006.

[12] E. Papadavid, J. Economidou, A. Psarra et al., "The relevance of peripheral blood T-helper 1 and 2 cytokine pattern in the evaluation of patients with mycosis fungoides and Sézary syndrome," British Journal of Dermatology, vol. 148, no. 4, pp. 709-718, 2003.

[13] E. J. Kim, S. Hess, S. K. Richardson et al., "Immunopathogenesis and therapy of cutaneous T cell lymphoma," The Journal of Clinical Investigation, vol. 115, no. 4, pp. 798-812, 2005.

[14] C. L. Berger, R. Tigelaar, J. Cohen et al., "Cutaneous T-cell lymphoma: Malignant proliferation of T-regulatory cells," Blood, vol. 105, no. 4, pp. 1640-1647, 2005.

[15] B. F. Chong, A. J. Wilson, H. M. Gibson et al., "Immune function abnormalities in peripheral blood mononuclear cell cytokine expression differentiates stages of cutaneous T-cell lymphoma/mycosis fungoides," Clinical Cancer Research, vol. 14, no. 3, pp. 646-653, 2008.

[16] T. Krejsgaard, L. M. Gjerdrum, E. Ralfkiaer et al., "Malignant Tregs express low molecular splice forms of FOXP3 in Sézary syndrome," Leukemia, vol. 22, no. 12, pp. 2230-2239, 2008.

[17] J. B. Heid, A. Schmidt, N. Oberle et al., "FOXP3 ${ }^{+}$CD $25^{-}$tumor cells with regulatory function in sézary syndrome," Journal of Investigative Dermatology, vol. 129, no. 12, pp. 2875-2885, 2009.

[18] T. Sasada, M. Kimura, Y. Yoshida, M. Kanai, and A. Takabayashi, "CD $4{ }^{+} \mathrm{CD} 25^{+}$regulatory $\mathrm{T}$ cells in patients with gastrointestinal malignancies: possible involvement of regulatory $\mathrm{T}$ cells in disease progression," Cancer, vol. 98, no. 5, pp. 1089-1099, 2003.

[19] T. J. Curiel, G. Coukos, L. Zou et al., "Specific recruitment of regulatory $\mathrm{T}$ cells in ovarian carcinoma fosters immune privilege and predicts reduced survival," Nature Medicine, vol. 10, no. 9, pp. 942-949, 2004.

[20] L. M. Gjerdrum, A. Woetmann, N. Odum et al., "FOXP3+ regulatory T cells in cutaneous T-cell lymphomas: association with disease stage and survival," Leukemia, vol. 21, no. 12, pp. 25122518, 2007.

[21] E. Capriotti, E. C. Vonderheid, C. J. Thoburn, M. Wasik, D. W. Bahler, and A. D. Hess, "Expression of T-plastin, FoxP3 and other tumor-associated markers by leukemic T-cells of cutaneous T-cell lymphoma," Leukemia and Lymphoma, vol. 49, no. 6, pp. 1190-1201, 2008.

[22] M. Kasprzycka, Q. Zhang, A. Witkiewicz et al., “ $\gamma c$-signaling cytokines induce a regulatory $\mathrm{T}$ cell phenotype in malignant $\mathrm{CD}^{+}$T lymphocytes," The Journal of Immunology, vol. 181, no. 4, pp. 2506-2512, 2008.
[23] E. A. Sausville, G. F. Worsham, M. J. Matthews et al., "Histologic assessment of lymph nodes in mycosis fungoides/sézary syndrome (cutaneous T-cell lymphoma): clinical correlations and prognostic import of a new classification system," Human Pathology, vol. 16, no. 11, pp. 1098-1109, 1985.

[24] E. C. Vonderheid, L. W. Diamond, W. A. van Vloten et al., "Lymph node classification systems in cutaneous T-cell lymphoma. Evidence for the utility of the working formulation of non-Hodgkin's lymphomas for clinical usage," Cancer, vol. 73, no. 1, pp. 207-218, 1994.

[25] G. Roncador, P. J. Brown, L. Maestre et al., "Analysis of FOXP3 protein expression in human $\mathrm{CD} 4{ }^{+} \mathrm{CD} 25^{+}$regulatory $\mathrm{T}$ cells at the single-cell level," European Journal of Immunology, vol. 35, no. 6, pp. 1681-1691, 2005.

[26] C. B. Harmon, "Detection of circulating T cells with $\mathrm{CD} 4^{+} \mathrm{CD} 7^{-}$ immunophenotype in patients with benign and malignant lymphoproliferative dermatoses," Journal of the American Academy of Dermatology, vol. 35, no. 3, part 1, pp. 404-410, 1996.

[27] M. Murphy, D. Fullen, and J. A. Carlson, "Low CD7 expression in benign and malignant cutaneous lymphocytic infiltrates: experience with an antibody reactive with paraffin-embedded tissue," American Journal of Dermatopathology, vol. 24, no. 1, pp. 6-16, 2002.

[28] K. E. Salhany, J. B. Cousar, J. P. Greer, T. T. Casey, J. P. Fields, and R. D. Collins, "Transformation of cutaneous T cell lymphoma to large cell lymphoma. A clinicopathologic and immunologic study," The American Journal of Pathology, vol. 132, no. 2, pp. 265-277, 1988.

[29] E. Diamandidou, M. Colome-Grimmer, L. Fayad, M. Duvic, and R. Kurzrock, "Transformation of mycosis fungoides/Sezary syndrome: Clinical characteristics and prognosis," Blood, vol. 92, no. 4, pp. 1150-1159, 1998.

[30] C.-D. Klemke, B. Fritzsching, B. Franz et al., "Paucity of FOXP3+ cells in skin and peripheral blood distinguishes Sézary syndrome from other cutaneous T-cell lymphomas," Leukemia, vol. 20, no. 6, pp. 1123-1129, 2006.

[31] T. Krejsgaard, N. Odum, C. Geisler, M. A. Wasik, and A. Woetmann, "Regulatory $\mathrm{T}$ cells and immunodeficiency in mycosis fungoides and Sézary syndrome," Leukemia, vol. 26, no. 3, pp. 424-432, 2012.

[32] G. J. Solomon and C. M. Magro, "Foxp3 expression in cutaneous T-cell lymphocytic infiltrates," Journal of Cutaneous Pathology, vol. 35, no. 11, pp. 1032-1039, 2008.

[33] T. Fujimura, R. Okuyama, Y. Ito, and S. Aiba, "Profiles of Foxp3+ regulatory $\mathrm{T}$ cells in eczematous dermatitis, psoriasis vulgaris and mycosis fungoides," British Journal of Dermatology, vol. 158, no. 6, pp. 1256-1263, 2008.

[34] Q.-A. Zhang, Z.-Q. Chen, M.-H. Chen, and Z.-D. Xu, “The number of regular $T$ cells and immature dendritic cells involved in mycosis fungoides is linked to the tumor stage," European Review for Medical and Pharmacological Sciences, vol. 18, no. 4, pp. 553-558, 2014.

[35] I. Fried and L. Cerroni, "FOXP3 in sequential biopsies of progressive mycosis fungoides," The American Journal of Dermatopathology, vol. 34, no. 3, pp. 263-265, 2012.

[36] D. A. Wada, R. A. Wilcox, R. H. Weenig, and L. E. Gibson, "Paucity of intraepidermal FoxP3-positive T cells in cutaneous T-cell lymphoma in contrast with spongiotic and lichenoid dermatitis," Journal of Cutaneous Pathology, vol. 37, no. 5, pp. 535-541, 2010.

[37] M. Alcántara-Hernández, C. Torres-Zárate, G. Pérez-Montesinos et al., "Overexpression of hypoxia-inducible factor 1 alpha 
impacts FoxP3 levels in mycosis fungoides-cutaneous Tcell lymphoma: clinical implications," International Journal of Cancer, vol. 134, no. 9, pp. 2136-2145, 2014.

[38] D. A. Wada, M. R. Pittelkow, N. I. Comfere, L. E. Gibson, S. M. Ansell, and R. A. Wilcox, "CD $4{ }^{+} \mathrm{CD} 25^{+} \mathrm{FOXP}^{+}$malignant $\mathrm{T}$ cells in Sézary syndrome are not necessarily functional regulatory T cells," Journal of the American Academy of Dermatology, vol. 69, no. 3, pp. 485-489, 2013.

[39] P. T. Walsh, B. M. Benoit, M. Wysocka, N. M. Dalton, L. A. Turka, and A. H. Rook, "A role for regulatory T cells in cutaneous T-cell lymphoma; induction of a CD4+CD25+Foxp3+ T-cell phenotype associated with HTLV-1 infection," Journal of Investigative Dermatology, vol. 126, no. 3, pp. 690-692, 2006.

[40] L. H. Shiue, X. Ni, V. G. Prieto et al., "A case of invisible leukemic cutaneous $\mathrm{T}$ cell lymphoma with a regulatory $\mathrm{T}$ cell clone," International Journal of Dermatology, vol. 52, no. 9, pp. 1111-1114, 2013.

[41] R. A. Clark, "Regulation gone wrong: a subset of sézary patients have malignant regulatory $\mathrm{T}$ cells," Journal of Investigative Dermatology, vol. 129, no. 12, pp. 2747-2750, 2009. 


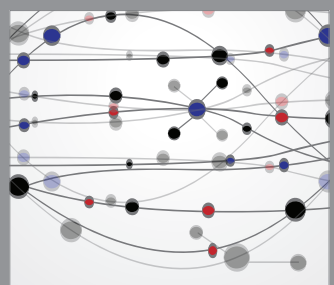

The Scientific World Journal
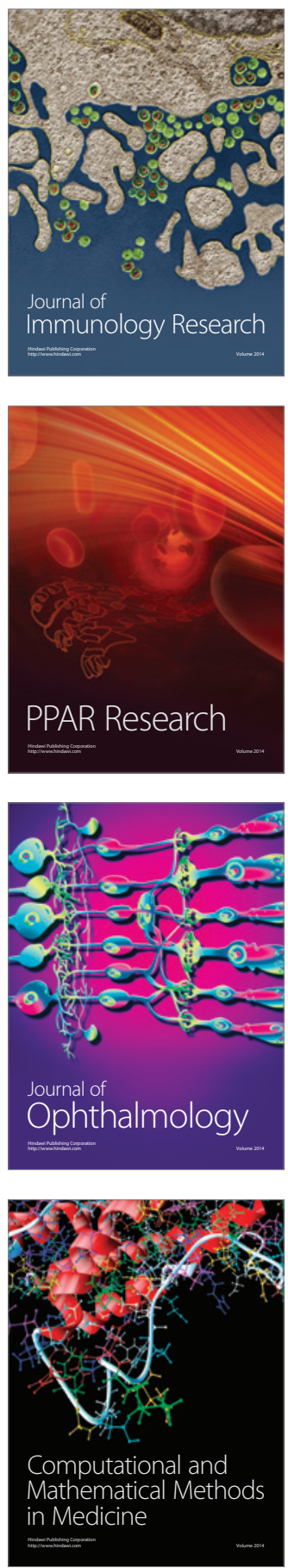

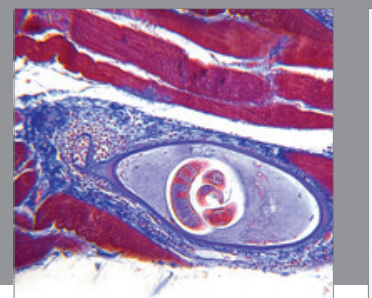

Gastroenterology

Research and Practice
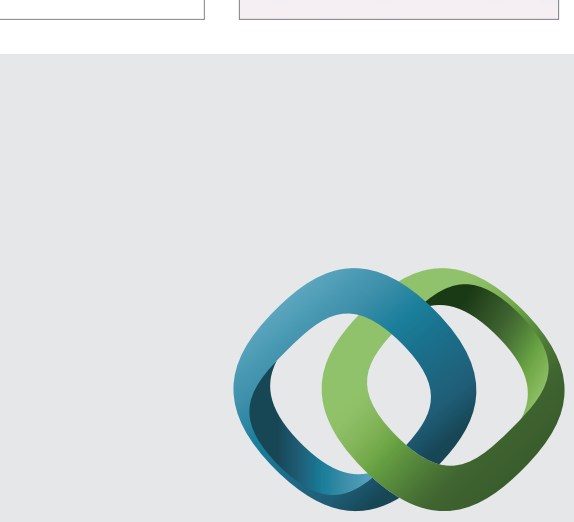

\section{Hindawi}

Submit your manuscripts at

http://www.hindawi.com
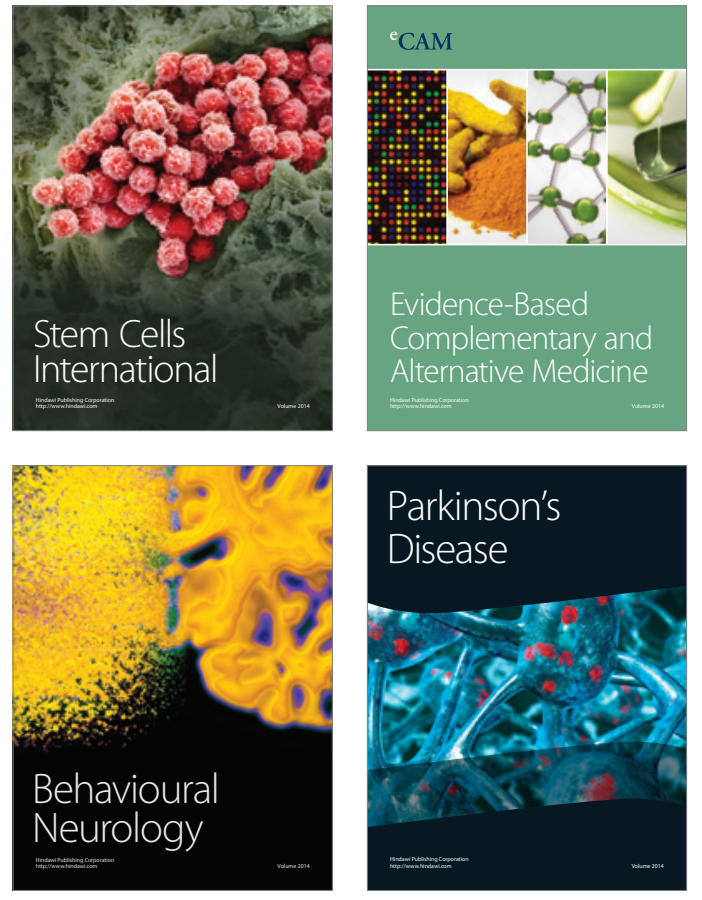
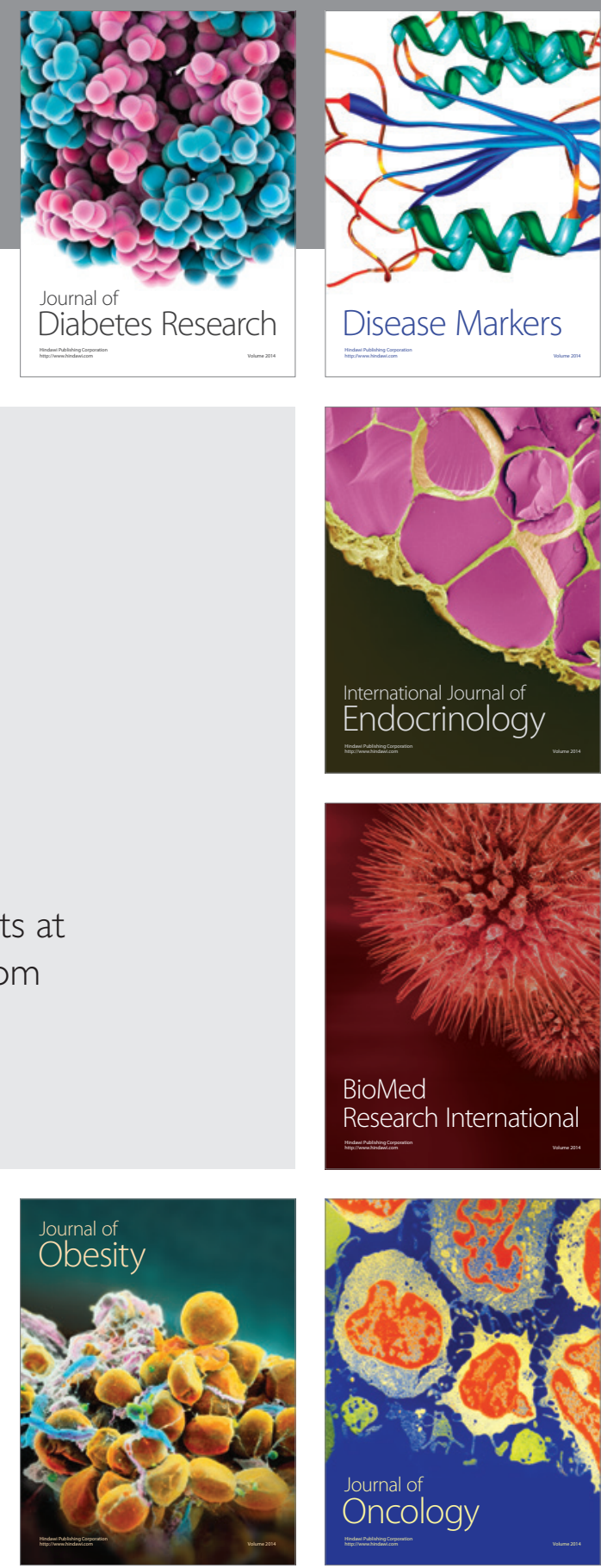

Disease Markers
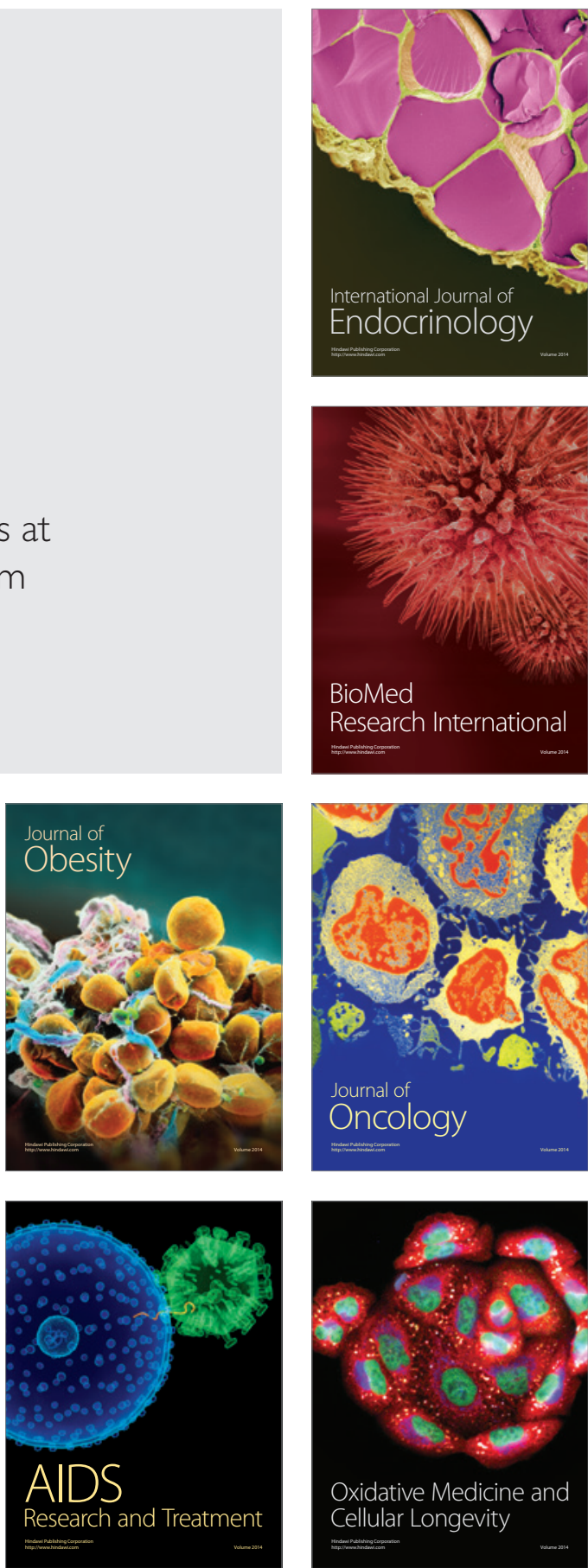